\title{
Temporal-contrast imperfections as drivers for ultrafast laser modifications in bulk silicon
}

\author{
Andong Wang $\odot,{ }^{*}$ Amlan Das, and David Grojo $\odot^{\dagger}$ \\ Aix-Marseille University, CNRS, LP3 UMR 7341, 13009 Marseille, France
}

(Received 13 February 2020; revised 18 May 2020; accepted 23 June 2020; published 6 July 2020)

\begin{abstract}
Despite recent successes in three-dimensional ultrafast laser writing inside silicon and other narrow gap materials, strong discrepancies remain in the identified narrow experimental windows to exceed material breakdown thresholds. In an experiment in which we irradiate silicon with perfectly synchronized femtosecond, picosecond, and nanosecond pulses, we show that the temporal contrast of the ultrashort pulses is a critical driving parameter, even more than the achievable peak vacuum intensity. We identify by appropriate pulse combinations breakdown channels seeded by pre-ionization and thermal runaway from the simulated contrast imperfections. The introduction of this multi-timescale control of the interactions allows bulk writing inaccessible otherwise. It also permits a comprehensive review of previous reports using infrared laser technologies at different characteristic contrast performances. The quantitative analysis of the problem reveals an extremely high sensitivity with modification ignition at intensity contrasts down to $10^{-6}$ and below. This sensitivity level is common for high-intensity physics experiments but has no equivalent in any other laser material processing application.
\end{abstract}

DOI: 10.1103/PhysRevResearch.2.033023

\section{INTRODUCTION}

Focused to small focal spots in transparent materials, intense femtosecond light pulses lead to local nonlinear energy deposition that makes possible to induce various microscale modifications anywhere in a three-dimensional (3D) space. In about two decades, this has led to the emergence of an alternative to lithography for direct microfabrication of microdevices inside dielectrics and unique techniques for precision surgery in tissues transparent to visible or near-infrared light [1,2]. With the advent of new sources directly emitting in the shortwave infrared (SWIR) region of the spectrum (1.1-2.5 $\mu \mathrm{m})$, one should see similar technologies applied to narrow gap materials as important as silicon but those are much slower in coming. This is because first attempts have faced abnormally strong requirements on the irradiation conditions in comparison to dielectrics. The explanation given today is strong detrimental nonlinearities intrinsic to narrow gaps and the use of long wavelengths. These include propagation phase distortions and losses caused by ionization and plasma effects developing prior to the focus and preventing in most cases enough space-time localization of laser energy to achieve permanent modifications [3-6].

Very recently, some solutions have been identified to circumvent these limitations [5-9] but it is striking to note

\footnotetext{
*andong.wang@univ-amu.fr

†david.grojo@univ-amu.fr
}

Published by the American Physical Society under the terms of the Creative Commons Attribution 4.0 International license. Further distribution of this work must maintain attribution to the author(s) and the published article's title, journal citation, and DOI. that different groups report very contradictory results for apparently similar experimental conditions. For a condensed presentation, the various results obtained using subpicosecond pulses are gathered in Table I mentioning the experimental conditions and observations for each study. In particular, one can note that the achievability of internal micromodifications in $\mathrm{Si}$ remains a large matter of debate. Some argues that modification regimes are inaccessible with conventional laser machining configurations because of the strong propagation nonlinearities delocalizing intense light [3-5]. Others have recently presented demonstration of waveguide writing in $\mathrm{Si}$ under conditions which are very similar to those used in dielectric studies $[9,10]$. As we will show in this paper, the controversy persists because all comparisons are made on the basis of the usual triplet of control parameters, namely the pulse duration, the pulse energy and the numerical aperture (NA) of the focusing lens (given in Table I). While this is usually appropriate with no apparent anomaly in the dependencies of the material responses to this triplet for surface or bulk dielectric interaction studies, we show in this paper that the temporal contrast, a laser-technology-dependent parameter, is a somewhat more important aspect to relate the observables inside narrow gap materials. The level of nonlinear delocalization of femtosecond pulses is actually so severe that it does not contribute more to ionization and energy transfer to the material than a picosecond pedestal or satellite pulse at intensity ratio as low as $10^{-3}$. With combined effects depending on synchronizations and associated ionization dynamics, this gives the possibility for internal structuring of $\mathrm{Si}$ that would be inaccessible otherwise with perfect-contrast pulses. We show this sensitivity to contrast imperfections reaches values below $10^{-6}$ when thermal runaway caused by a long background signal is the driver and identify in this way the real interaction dynamics in previous reports. 
TABLE I. Summary of laser conditions and observations in studies aiming at permanent modification in bulk silicon with tightly focused subpicosecond pulses. The references are sorted chronologically mentioning the pulse duration $\tau$, the wavelength $\lambda$, the maximum pulse energy $E_{\max }$, the repetition rate and the numerical aperture NA used for focusing. The laser technology used for the experiments as well as the

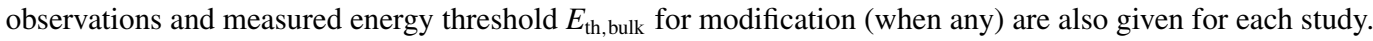

\begin{tabular}{|c|c|c|c|c|c|c|c|c|}
\hline $\begin{array}{l}\tau \\
(\mathrm{fs})\end{array}$ & $\begin{array}{c}\lambda \\
(\mu \mathrm{m})\end{array}$ & $\begin{array}{l}E_{\max } \\
(\mu \mathrm{J})\end{array}$ & $\begin{array}{l}\text { Rep. rate } \\
(\mathrm{kHz})\end{array}$ & NA & $\begin{array}{l}\text { Laser } \\
\text { technology }\end{array}$ & Observations & $\begin{array}{c}E_{\mathrm{th}, \text { bulk }} \\
(\mu \mathrm{J})\end{array}$ & Ref. \\
\hline 70 & 2.4 & 1.7 & 1 & 0.5 & OPA & $\begin{array}{c}\text { Near-surface modification only; } \\
\text { Positive index change (waveguide) }\end{array}$ & - & {$[11]$} \\
\hline 250 & 1.2 & 90 & 1 & 0.2 & OPA & Ionization but no bulk modification & - & {$[3]$} \\
\hline 110 & 1.24 & 600 & 1 & 0.85 & $\begin{array}{l}\text { Cr:Forsterite } \\
\text { Regen. }\end{array}$ & No Bulk modification & - & {$[7,12]$} \\
\hline 800 & 1.55 & 50 & 100 & 1.25 & Er-doped fiber & Bulk modification & $<50$ & {$[13]$} \\
\hline 100 & $1.3-2.2$ & 1 & 1 & 0.3 & OPA & $\begin{array}{c}\text { Measurable NL absorption but } \\
\text { no bulk modification }\end{array}$ & $<50$ & {$[14,15]$} \\
\hline 60 & 1.3 & 10 & 0.1 & $0.3-2.2$ & OPA & $\begin{array}{l}\text { Saturated microplasma and fluence; } \\
\text { No bulk modification }\end{array}$ & - & {$[4,5,16]$} \\
\hline 100 & 1.3 & 12 & 1 & 0.4 & OPA & $\begin{array}{c}\text { No bulk modification } \\
\text { with high-angle Bessel beam }\end{array}$ & - & [17] \\
\hline 60 & 1.3 & 1000 & 100 & 0.3 & OPA & $\begin{array}{l}\text { No bulk modification } \\
\text { with high-energy Bessel }\end{array}$ & - & {$[18]$} \\
\hline $\begin{array}{l}110 \\
\text { (double) }\end{array}$ & 1.24 & 55 & 1 & 0.85 & Cr:Forsterite & & & \\
\hline 250 & $1.2-5.25$ & 10 & - & 0.3 & $\begin{array}{l}\text { Regen. } \\
\text { Simulation }\end{array}$ & $\begin{array}{c}\text { Bulk modification } \\
\text { Wavelength-independent } \\
\text { saturation of excitation below breakdown }\end{array}$ & $>10$ & {$[7]$} \\
\hline 60 & 1.3 & 20 & $0-100$ & 3 & OPA & $\begin{array}{l}\text { Bulk modification; } \\
\text { Negative index change (measurement) }\end{array}$ & $<0.02$ & {$[5]$} \\
\hline 350 & 1.55 & 2 & 250 & 0.5 & Er-doped fiber & $\begin{array}{l}\text { Continuous bulk writing; } \\
\text { Positive index change (waveguide) }\end{array}$ & $2^{\mathrm{a}}$ & [9] \\
\hline 800 & 1.55 & 6.7 & 200 & 0.45 & Er-doped fiber & Bulk Modification (stochastic) & 6 & [8] \\
\hline 800 & 1.55 & 10 & 400 & 0.45 & Er-doped fiber & $\begin{array}{c}\text { Continuous bulk writing; } \\
\text { Positive index change (waveguide) }\end{array}$ & $\approx 0.07^{\mathrm{a}}$ & {$[10]$} \\
\hline 200 & 1.24 & 5 & 0.01 & 0.5 & $\begin{array}{l}\text { Cr:Forsterite } \\
\text { Regen. }\end{array}$ & No bulk modification & - & {$[20]$} \\
\hline
\end{tabular}

$\overline{\bar{a} \text { The modification is seeded by precursor defects found, for instance, at sample surfaces. The authors mention an incapacity on obtaining a }}$ systematic modification that could be created anywhere inside pure Si crystals in these works.

\section{RATIONALE}

Expanding on the strong contradictions between previous reports, we illustrate with colored boxes in Fig. 1(a) the identified sets of inappropriate parameters to achieve bulk modifications with subpicosecond laser pulses. The results are shown as function of NA for focusing and pulse energy without considering the wavelength. This is because most studies are conducted in a range $(1.2-1.55 \mu \mathrm{m})$ corresponding to two-photon absorption initiated interactions in $\mathrm{Si}$ (band gap $E_{g}=1.1 \mathrm{eV}$ ) and the other studies have concluded on a very modest dependence to this parameter [14,19]. On the graph, the red region shows a large set of effective numerical aperture (NA) and pulse energy combinations for which bulk modification inside silicon is claimed inaccessible on the basis of experiments carried out with femtosecond laser sources converted to the infrared by optical parametric amplification (OPA). In brief, the red box includes NAs up to 2.3 [5] and pulse energies tested up to the millijoule level [18] with $\approx 100$ fs pulses at 1300 -nm wavelength (single and multishot conditions). Only extreme focusing conditions exploiting solid-immersion schemes to achieve effective NA near 3 have been successful in achieving modifications in these experiments [5]. Interestingly, very modest energy become however required $[\approx 10 \mathrm{~nJ}$; see red solid circle in Fig. 1(a)] in this case revealing the limitation on the conditions applied to matter is primarily caused by the geometry of the energy flux. This is also consistent with earlier works including Bessel beam irradiation $[17,18]$ and pump-probe studies $[3,4]$ reporting excitations of Si clamped well below material breakdown because of the relatively modest focusing geometry implemented in these experiments (effective NA up to 0.45). However, as shown by all solid symbols in the red region of Fig. 1(a) some works remain in total contradiction to this conclusion. For instance two groups have presented evidence of waveguide writing conditions with compact Er:doped fiber laser technologies directly emitting at similar wavelength. The positive refractive index change reported in these works is obtained with NA and pulse energies well below the requirements associated to the OPA studies $[9,10]$. Since those reported conditions for seed modifications and controlled writing do not significantly exceed the threshold for modification (typically $5 \mu \mathrm{J}$ and NA $\approx 0.5$ ), they define another window shown in blue with the most obvious disagreement to the OPA studies. 

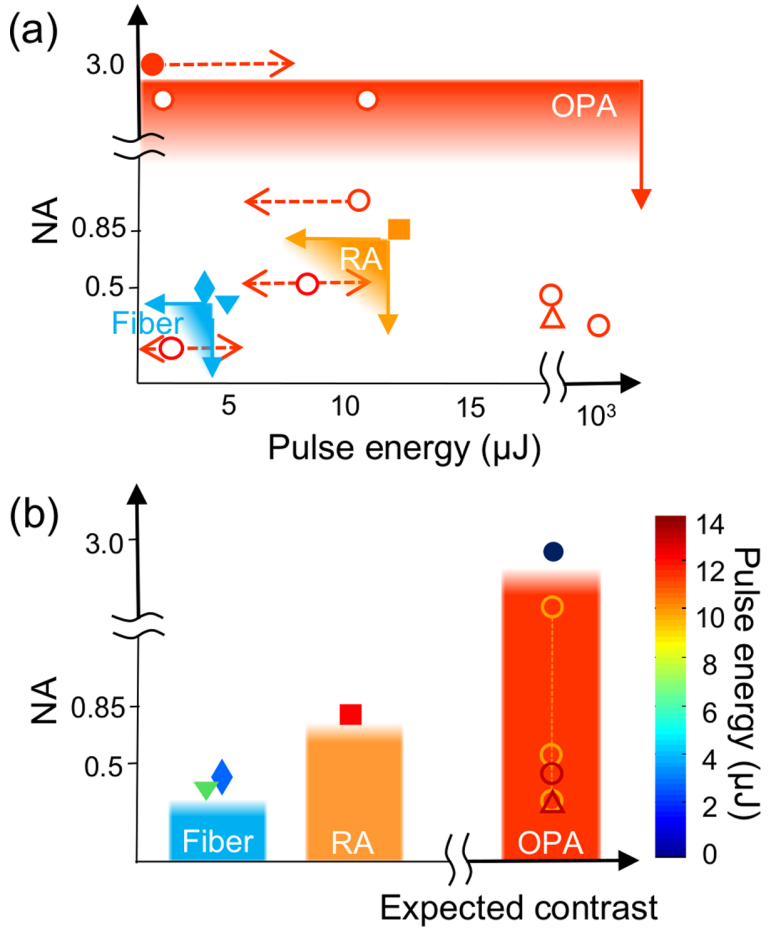

FIG. 1. Comparison of modification attempts in bulk Si using subpicosecond laser pulses. The empty symbols indicate unsuccessful attempts while solid symbols show conditions for which permanent modification is reported. (a) Outcome of the tested conditions as function of NA and pulse energy. The symbol colors refers to the different type of laser technologies. The colored regions (see also arrows) show the strong difference between the inappropriate parameter regions depending on technologies. (b) Same data but sorted by technologies. Symbol colors refers to the applied pulse energy level and the results are displayed as function of NA and expected temporal contrast depending on technologies. The introduction of contrast solves the apparent contradictions in (a). Data are extracted from: our previous works (०) $[4,5,17,18]$, and Kononenko et al. $(\triangle)$ [3] using optical parametric amplification (OPA), from Shimotsuma et al. ( $\square$ ) [7] using a Cr:F amplified laser (RA), and from Nolte et al. ( $\nabla)[8,10]$ and Pavlov et al. ( $\diamond)[9]$ using Er:doped fiber lasers.

In view to these first remarks, one can directly envision that the conclusions are laser-technology related. This leads us to highlight here major differences between pulses generated by OPA and fiber technologies. There are unique advantages to using Er:doped fiber laser technologies because they allow compact systems directly emitting in the appropriate SWIR region (1550 $\mathrm{nm}$ in these works) and repetition rates inaccessible with other technologies. With unprecedented repetition rates up to $400 \mathrm{kHz}$ some previous works (see Table I) have concluded that the successful modifications with these technologies were based on accumulation $[9,10]$. However, a timescale analysis makes this hypothesis debatable as even higher repetition rates are required in principle to rely on free-carrier or heat accumulation on a shot-to-shot basis in silicon [21]. Interestingly, we identify here that another specificity of fiber sources lies on the temporal contrast. Their developments rely on difficult dispersion management for re-compression due to accumulated nonlinear propagation effects (e.g., self-phase modulation) in amplifying fibers [22]. As we will see latter on, we found in all the autocorrelation traces in the literature (some corresponding to lasers referenced in Table I $[8,13]$ ) a significant picosecond pedestal. This is a direct evidence of incomplete compression but we can also add that the developments often refer to a typical $90 \%$ of the energy in the picosecond centroid in best cases [22]. At the opposite, one can reasonably assume a nearly perfect temporal contrast for pulses from femtosecond OPAs as the generation of pulses at microjoule level or more in these studies rely on several stages of optical parametric amplification, a second order nonlinear interaction process that efficiently cleans any potential pedestal or satellite pulses of the femtosecond pumping source [23].

We can extrapolate from this that higher is the temporal contrast stronger are NA requirements to achieve enough energy density in $\mathrm{Si}$ for modification. Interestingly, this is also supported by another work by Shimotsuma et al. [7] using a third type of laser technology. This study reports modification at $\mathrm{NA}=0.85$ and double-pulse energy of $\approx 12$ $\mu \mathrm{J}$ with a $\mathrm{Cr}$ :Forsterite amplified laser for direct emission at 1240-nm wavelength. One can assume that the temporal contrast for this source is similar to that known for Ti:Sapphire technology because of a similar solid-state design. The latter are associated with the amplified spontaneous emission (ASE) creating nanosecond background or satellite pulses that can occur in various ways (e.g., via imperfect switching of the Pockels cell or parasitic reflections in the regenerative amplifier) unless specific solutions are implemented to enhance the contrast (e.g., an additional pulse picker to suppress pre- and postpulses). This describes the causes of an imperfect temporal contrast for the technology but contrast values largely exceeding 1:100 and negligible energy in satellite pulses are readily obtained. This is confirmed by the need of third order cross-correlators to detect the small pre- or postpulses as only the main pulse can be measured by second-order autocorrelators. Overall, this corresponds to an intermediate contrast between fiber lasers and OPA and it is interesting to note in Fig. 1(a) that the NA and energy requirements for modifications are positioned between (orange box).

To illustrate our view of a laser-technology dependent problem, we present in Fig. 1(b) the same data as in Fig. 1(a) but looking at the different technologies separately, ordered on the horizontal axis as function of expected temporal contrasts. First, we see that most of contradictions are solved once we look at the results for each technology separately. However more important is that we observe also a clear trend with the expected contrast leading to the conclusion that higher is the temporal contrast of the ultrashort pulses stronger are the NA requirements to exceed the modification threshold.

The aim of this paper is thus to demonstrate the validity of this conclusion. We design and perform an experiment for a quantitative analysis of the problem. By synchronization of picosecond and nanosecond pulses with a highly contrasted pulse from an OPA, we control the temporal characteristics of irradiation at different timescales and degrade the apparent contrast. By reproducing the general characteristics of the previously mentioned laser technologies, we confirm an unusual level of sensitivity to the contrast for laser processing and differentiate the physical effects between pre- and postpulses. 


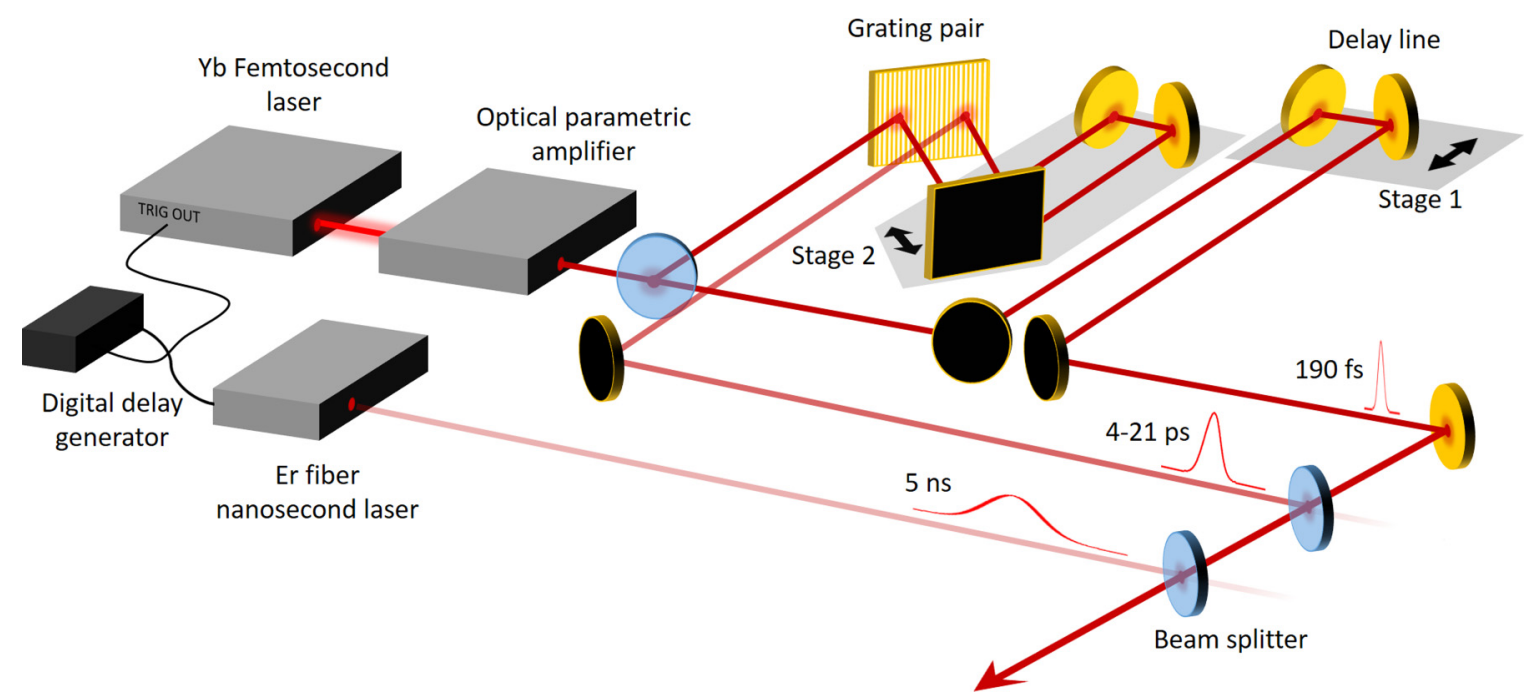

FIG. 2. Simplified schematic of the experimental arrangement used for multi-time scale control of infrared pulses. An amplified Ytterbium femtosecond laser is used as master source. The beam pumps an optical parametric amplifier to generate pulses of about $190 \mathrm{fs}$ at 1550 -nm wavelength. A beam splitter is used to create a separated picosecond laser beam with a grating-based stretcher arrangement. A motorized delay line (stage 1) is implemented for synchronization of the femtosecond and picosecond pulses. In the stretcher, one grating and retro-reflector can be moved (Staged 2) to continuously change the pulse duration from 4 to $21 \mathrm{ps}$. A synchronization signal from the femtosecond laser is used to trigger an erbium-based fiber laser emitting 1550-nm pulses of 5-ns duration at delay that can be adjusted electronically. The combination of all pulses using thin-film optics create a new beam directed toward the bulk-Si interaction setup (not shown).

\section{EXPERIMENTAL SETUP}

In the experiments, we rely on the synchronization of engineered pulses for multi-timescale analysis of the material response dependency to the temporal contrast. We show on a simplified schematic given in Fig. 2 the implemented optical elements for controlling the temporal characteristics of irradiation. We use a femtosecond laser source (Pharos, Light-conversion) generating $500-\mu \mathrm{J}$ pulses at $1030-\mathrm{nm}$ central wavelength. Then, the beam is converted to $1550 \mathrm{~nm}$ by using an OPA (Orpheus, Light-conversion) delivering pulses of sub-190-fs duration (FWHM as measured by autocorrelation), that is on the order of the shortest pulses used in the context of internal Si structuring (see Table I). For the pump laser, a prepulse contrast $<1: 1000$ and a postpulse contrast $<1: 200$ are specified as measured with a photodiode. These translates into extremely high contrasts after the OPA, basically zero, because of a white light generation threshold far above pre/postpulse intensity and the use of three stages of optical parametric amplification for efficient conversion energy transfer to the 1550 -nm wavelength. The only imperfections that we can envision are ultrashort postpulses caused by some internal reflections but in any case far below 1:1000 according to the absence of detectable signature is all signals shown hereafter.

To artificially degrade and adjust the picosecond contrast of the pulse, we split the beam to inject a portion in a stretcher arrangement with two Littrow gratings introducing negative group velocity dispersion to the original laser pulses. The details of the configuration are discussed elsewhere [24]. However, it is important to note here that the distance between the gratings (see stage 2 on Fig. 2) can be changed to tune the duration of the stretched pulses from 4 to 21 ps. This combined to a delay line inserted into the path of the the main femtosecond laser beam (see stage 1 on Fig. 2) makes possible to simulate picosecond prepulse, postpulse contribution as well as incompletely recompressed pulses depending on technolgies (e.g., fiber lasers) as discussed before.

Because the relevant timescale to define the contrast of ultrashort pulses can extend to the nanosecond range (e.g., ASE contribution), we also synchronize a $10-\mu \mathrm{J}$ class nanosecond laser source (MWTech, PFL-1550) which generates pulses at $1550 \mathrm{~nm}$ wavelength. The pulse duration is $5 \mathrm{~ns}$ (FWHM). It is externally triggered using the femtosecond source as clock and the delay between the nanosecond and femtosecond pulses are changed ( $\pm 100 \mathrm{~ns}$ ) with $25 \mathrm{ps}$ precision by simply using a digital delay generator (Standford DG645).

Another aspect of major importance and not shown in Fig. 2 is that each beam line is equipped with a half-wave plate and polarizer combination so that all beams contributing to the irraditions have identical linear polarization and the energy contribution of each pulse defining the contrast at different delays can be continuously adjusted. Another important detail is the use of a telescope in the beam path of the nanosecond laser to obtain three beams of identical size.

The colinearity of the three beams after recombination using thin-film beam splitters as shown in Fig. 2 is made using two irises with $>1-\mathrm{m}$ separation. Then, for interaction studies, the three-component beam is tightly focused using a 0.85 NA microscope objective (Olympus LCPLN-IR 100×) with spherical aberration compensation. A high-resolution imaging system is also used to guarantee nearly perfect spatial overlap of the focused spots from the three components. The prepared tree-component pulses are focused $300 \mu \mathrm{m}$ below the surface of $\mathrm{Si}$ samples. The samples are 1-mm thick intrinsic Si crystals with $100\left( \pm 5^{\circ}\right)$ orientation. The lasers are operated at $1-\mathrm{kHz}$ 
repetition rate and a fresh region of the sample systematically exposed during $2 \mathrm{~s}$ for comparison of the material response depending on the other temporal irradiation characteristics. We make this choice because irradiation with repeated 2000 pulses allow to develop modification which can be readily observed with $20 \times$ magnification infrared imaging. The latter consists in the assembly of a microscope objective, a tube lens and an InGaAs array detector behind the sample for backside in situ detection of the potential micro-modifications. For each temporal contrast condition, the bulk modification energy threshold (if so) is estimated by irradiating fresh regions of the sample with gradually decreasing pulse energies until no modification is observed. Then, the precision that will be shown by error bars are directly depending on the energy steps used in each procedure.

\section{RESULTS AND DISCUSSION}

\section{A. Autocorrelation signal measurements}

Before each interaction experiment, the temporal characteristics of the prepared pulses are systematically verified. We use a home-made second-harmonic ( $\mathrm{SH}$ ) intensity autocorrelator (AC). A long delay line on this system allows a measuring range of $160 \mathrm{ps}$ with a resolution of $25 \mathrm{fs}$. With the acquired traces, as shown in Fig. 3, we can access to the contrast intensity ratio, the delay between the picosecond and femtosecond components as well as the duration of the picosecond pulses which are used. Obviously the intensities of the nanosecond pulses are too low to be detected together with the ultrashort pulses in a SH autocorrelator but the delay to the femtosecond pulse is systematically checked with subnanosecond precision by using a fast photodiode and an oscilloscope.

To show the level of control on the contrast characteristics of the irradiating pulse with the multibeam combination, we show in Fig. 3(a) autocorrelation traces obtained with the combination of our 190 fs laser pulse with a stretched pulse at 11 ps (FWHM). The two pulses are synchronized (zero delay) and the intensity ratio between the two pulses is varied from 1:6 to $1: 100$ by independent control of pulse energy on each beam line. We note from these measurements that from a contrast of $1: 100$, it becomes almost impossible to reveal the picosecond background contribution. This is an important aspect here because it defines a contrast level high enough so that the imperfections are not detectable (unless high dynamics diagnostics are used) but, as we will show later too low to avoid an important contribution of the background in the Si modification response. With Fig. 3(a), we also note an excellent agreement obtained between the AC signal measurements (black curves) and the theory assuming Gaussian temporal shapes for both pulses (see red curves). This shows the knowledge on the irradiation conditions and the possibility to extrapolate from this measurement all accessible changes on the contrast characteristics by continuously varying the intensity ratio, the delay between pulses and the picosecond pulse duration to create different pre-, postpulses, or pedestal [with zero delay as shown in Fig. 3(a)] for investigation of the contrast dependence of this problem.

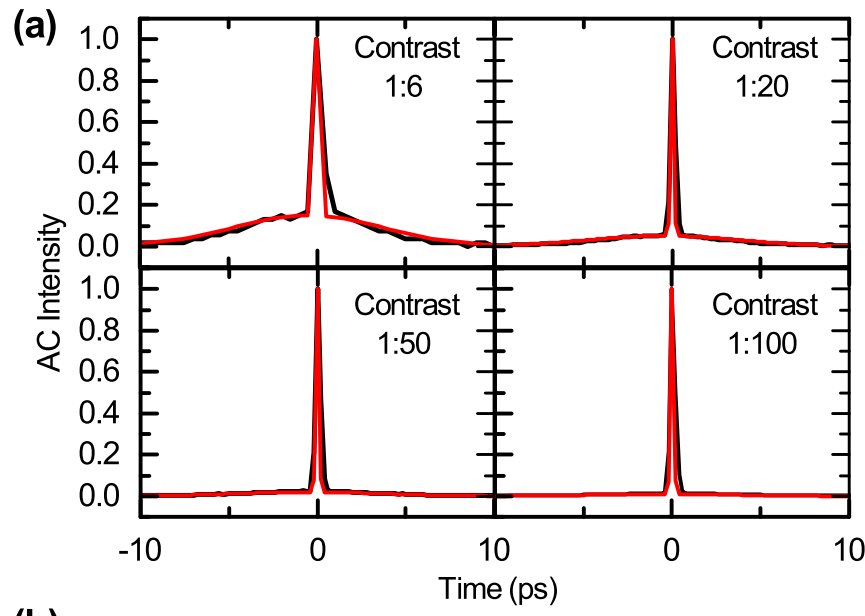

(b)

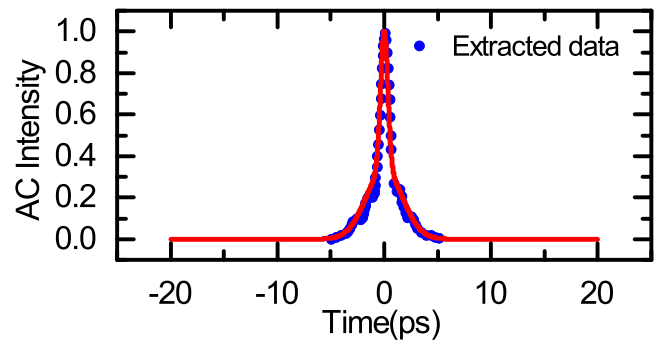

FIG. 3. Autocorrelation (AC) measurements for pulses prepared with different contrasts (a). The black solid line shows experimental results and the red solid line is a theoretical curve assuming the combination of two Gaussian pulses with durations of $190 \mathrm{fs}$ and $11 \mathrm{ps}$, a perfect synchronization (zero delay) and the intensity ratio mentioned on each graph. (b) Autocorrelation measurement results extracted from Ref. [22] for the fiber system used in several studies reporting bulk modification in $\mathrm{Si}[8,10,13]$. The data are compared to the same fitting procedure identifying a 800 -fs main pulse and a 4-ps pulse pedestal.

Finally, to show a range of accessible parameters relevant for comparative studies with cases listed in Table I, we have extracted as an example the data of Ref. [22] giving an autocorrelation trace for the Er:doped fiber laser used in Si modification studies $[8,10,13]$. These are shown with the blue spots in Fig. 3(b). Despite a pulse as short as $800 \mathrm{fs}$ (FWHM), it reveals an important background that can be directly identified between -5 and $5 \mathrm{ps}$. As we show with the red curve, we can model this by a 4-ps pulse pedestal using an intensity as low as 2:1. Interestingly, this is in the range of accessible parameters with our setup. With the OPA, we can thus perform experiments with conditions which are relatively similar to those of the papers using fiber lasers and reporting bulk modifications in $\mathrm{Si}$.

An additional comment is that the zero signal outside the 10 ps range in the autocorelation trace of Fig. 3(b) does not imply a total absence of energy that may contribute as we discussed before. More precisely, the specifications of the commercial fiber laser system considered here guarantee $90 \%$ of energy in a 10-ps range. This potentially leads to an added complication to the problem but that we can address with the synchronization of the nanosecond pulse in our experiment to simulate the typical $10 \%$ remaining energy distributed on a longer time scale. 


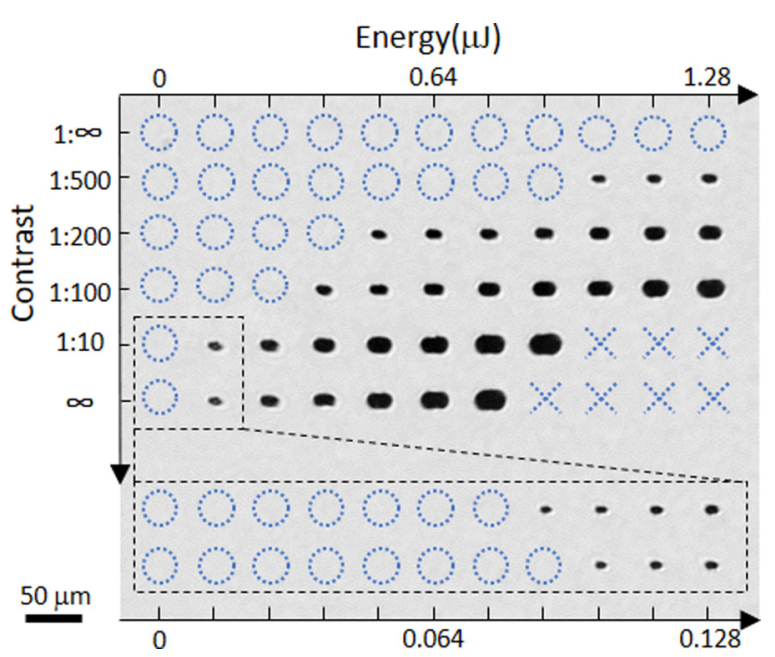

FIG. 4. Bulk Si modifications obtained after irradiation with 190 -fs pulses and picosecond prepulse contrasts of different energies on separated sites. The duration of the prepulse degrading the contrast is $10 \mathrm{ps}$. The impacts are made $300-\mu \mathrm{m}$ below the surface and observed with infrared microscopy. The blue circles indicates irradiations without modifications and crosses are cases in the matrix that have not been tested because of laser energy limitations. We note the incapacity of achieving modification with high-contrast femtosecond pulses and a contrast-dependent modification threshold given by the transition between the last circle and the first modification on each line. Scale bar is $50 \mu \mathrm{m}$.

\section{B. Contrast-dependent bulk modification thresholds}

For direct evidence of the influence of the temporal contrast for internal Si structuring, we show in Fig. 4 a top view of a typical matrix of laser impacts in bulk $\mathrm{Si}$ as prepared for quantitative analysis. The matrix shows by infrared microscopy the result of irradiations repeated on fresh regions separated by $50 \mu \mathrm{m}$ inside the sample with a varying pulse energy from 0 to $1.28 \mu \mathrm{J}$ depending on position on the horizontal axis and different contrasts depending on position on the vertical axis. In this experiment, the contrast is varied by proper energy combination of a 11-ps pulse and a 190-fs pulse (main pulse) delayed by 20 ps so that we simulate a situation with progressively degraded picosecond prepulse contrast. While we have measured the influence of the delay between pulses (not shown), we have chosen to keep the delay at 20 ps for all data presented on the picosecond contrast in this paper (positive or negative). This reasonably simplifies the presentation while it leads to a maximum of cross-action between pulses. We found this is particularly true for the postpicosecond pulse cases for which we found decreasing combined effects for longer delays that can be attributed to a fast decay of the plasma created by the femtosecond pulse [16].

In Fig. 4 comparing different prepulse contrast values, we show also the material response with a "pure" femtosecond irradiation and a "pure" picosecond irradiation obtained by simply blocking one of the beam lines (see Fig. 2). First, we confirm the incapacity of inducing any modification with the high-contrast femtosecond pulse alone due to the nonlinear delocalization of light as reported with other studies conducted with OPAs $[3,4]$. The underlying mechanisms include progressive and strong two-photon absorption before the focus as well as plasma screening or scattering effects preventing the pulse energy to be efficiently delivered near focus. The pure picosecond case interestingly shows that stretching the pulse to the picosecond domain solves this limitation [6]. An energy threshold for modification as low as $\approx 100 \mathrm{~nJ}$ is found (" $\infty$ " contrast line in Fig. 4). This shows that (i) reduced beam peak intensities (and so delocalization) and (ii) potential assistance of energy deposition by band gap closure when pulse duration exceeds electron-phonon coupling time gives a way to circumvent the clamping on the delivered energy density. This is a very modest energy in comparison to those tested in various subpicosecond pulse studies shown in Table I. Accordingly, it opens the door for a possible modification seeded by prepulse or pedestal in case of imperfect contrast in the previously reported successful cases.

This is actually directly evidenced by comparison of the energy-dependence response that varies with the contrast in Fig. 4. For a femtosecond irradiation with a contrast of $2 \times$ $10^{-3}$, we note that a modification can be initiated with the low energy pulses tested in this particular experiment. In these observations, we concentrate on modest contrasts and energies so that both components contributes to energy deposition but we directly understand from the material response that the possibility to achieve modification holds also for much higher contrast values as the prepulse can always induce a modification by itself, provided that the applied total energy is sufficiently high. In the tested range of conditions, we observe modifications with apparent lateral dimension varying from about $7 \mu \mathrm{m}$ to $>20 \mu \mathrm{m}$. Another observation with Fig. 4 is a dissymmetry in the produced features that is not visible with longer pulses (e.g., nanosecond pulses used hereafter). Their orientation is polarization-dependent as observed by others with double-pulse irradiation under similar focusing conditions. While the underlying mechanisms behind this observation is an intriguing aspect that will deserve more investigations, we concentrate here on the differences in energy thresholds for all tested contrasts in this experiment. In comparison to the 'pure' picosecond case, the threshold can be slightly lower (low contrast, see bottom lines) evidencing increased efficiency by combined contributions or higher for high contrasts leading to a situation somehow worse than the 'pure' picosecond case but still providing enough assistance for the femtosecond pulse to achieve modifications that are inaccessible with highly contrasted pulses. Taken together these observations illustrate the high sensitivity of the results of these experiments to the contrast.

For a more quantitative analysis of this case, Fig. 5 reports the measured energy thresholds as function of intensity contrast ratios. For comparison the measured threshold with the picosecond pulse (alone) is shown on the same graph with a red energy level. The graph confirms that a contrast on the order of $10^{-1}$, that is interestingly typical for fiber lasers (see before) leads to a threshold which is lower than the pure picosecond case. This evidences combined physical effects leading to a more favorable situation to achieve modification with this technology. When increasing the contrast, it is striking to observe a drastic increase of the threshold (note the logarithmic scale). At $\approx 10^{-3}$, the measured threshold is one order of magnitude higher and not measurable for higher values due to an energy limitation at the microjoule level 


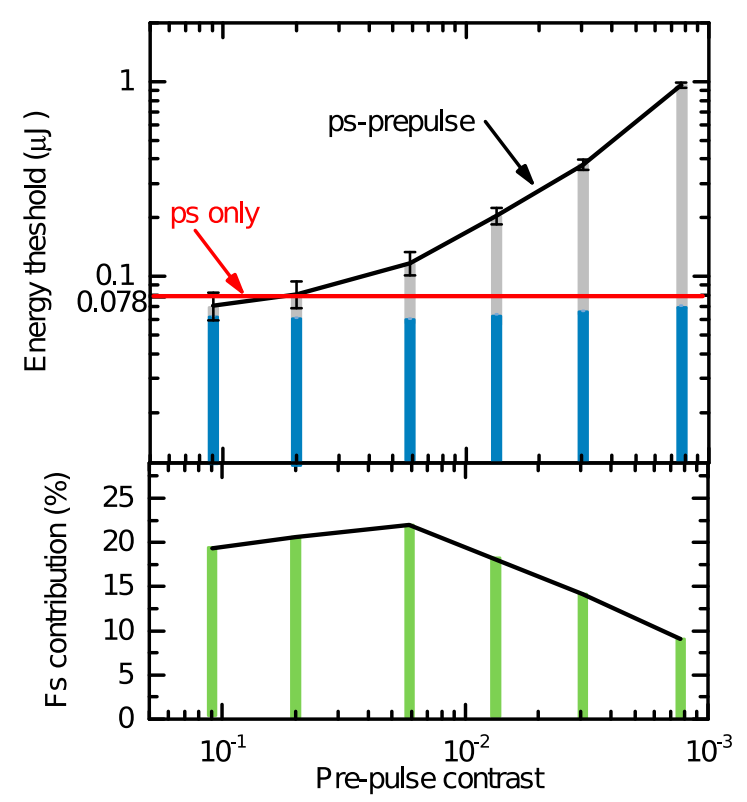

FIG. 5. Measured energy threshold for ultrafast laser induced modification in bulk silicon as function of prepulse contrast. The contrast is given by the peak intensity ratio of a 11-ps prepulse and the main 190 -fs delayed by 20 ps. In the upper part of the figure, blue and grey bars represent the applied energy with the picosecond and femtosecond pulse respectively. The relative contribution of the femtosecond pulse leading to modification, obtained by subtracting the threshold for the pure picosecond case shown in red to the measured threshold, is shown with linear scale in the lower part.

in this experiment. Beyond the possibility to achieve modification in any imperfect-contrast case at threshold directly depending to the contrast, the data also indicates a contribution of the femtosecond main pulse in all tested situations. This contribution can be directly extracted from the data by subtracting the applied picosecond pulse energy (first pulse, blue bars) to the measured threshold. It leads to the bottom graph of Fig. 5, where we note there is a contrast value of $\approx 5 \times 10^{-2}$ for a maximum relative contribution of the femtosecond pulse. Above this optimum, we observe that the femtosecond pulse contribution is progressively vanishing with high contrasts.

Whereas an individual femtosecond pulse cannot achieve modification, we see it can contribute to more than $20 \%$ of the energy deposition leading to modification in this experiment. This identifies conditions of major importance on which should concentrate future works because a consequence is that we can reasonably expect some differences in modifications in comparison to those achieved with high-contrast picosecond lasers. The spatial aspects of excitation probably play a large role in the optimum combination but we assume at this stage it relies on an appropriate heating and/or plasma density created by the picosecond pulse to seed efficient local absorption of the femtosecond pulse. As the delay is fixed in this experiment, the ability of the picosecond pulse to prepare ideally the material is maintained independently on the femtosecond pulse intensity. Accordingly, the decreasing contribution of the femtosecond pulse at high contrast can be reasonably attributed to a growing importance of detrimental plasma effects

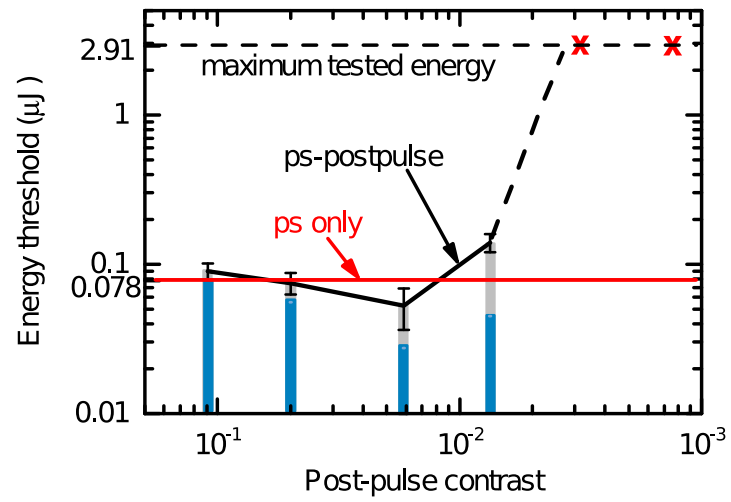

FIG. 6. Measured energy threshold for ultrafast laser induced modification in bulk silicon as function of prepulse contrast. The contrast is given by the peak intensity ratio of a main 190-fs pulse and 11-ps postpulse delayed by $20 \mathrm{ps}$. The blue and grey bars represent the applied energy with the picosecond and femtosecond pulse respectively. The measured threshold for pure picosecond irradiation is shown in red. The red crosses represent no modification with the maximum tested energy.

in the femtosecond interactions. At contrast values higher than $5 \times 10^{-2}$, the femtosecond pulse intensity becomes so high that it creates a strong plasma that delocalizes itself before reaching the focus by the same way it has been observed in single pulse previous studies [5]. The femtosecond pulse cannot contribute simply because it is hardly reaching the region prepared by the prepulse. This vision is fully consistent with an asymptotic behavior toward no net contribution of the femtosecond pulse for increasing contrasts (Fig. 5).

The optimum combined effect introduces the concept of femtosecond laser induced modification seeded by picosecond prepulse, a case that can be directly compared to the analog picosecond postpulse contrast response. The result of a similar analysis made by simply changing the delay from 20 to $-20 \mathrm{ps}$, that is swapping the picosecond and femtosecond pulses on the temporal axis but keeping all other parameters unchanged, is shown in Fig. 6. First, we note obvious differences between the graphs of Figs. 5 and 6 . This confirms directly that we are not investigating cases for which each individual pulse interacts independently. A main difference with this picosecond postpulse case is that we cannot directly evaluate the relative contribution of each pulse to energy deposition leading to modification because there is no energy threshold that can be reached with the first femtosecond pulse (alone) in this case. Also, we cannot compare the measurements to the measured threshold for the pure picosecond pulse because the femtosecond pulse affects the picosecond interaction in this case. However, an advantage with this situation is that femtosecond interactions inside $\mathrm{Si}$ has been investigated with time-resolved diagnostics in previous works $[4,16]$. These studies indicate that the picosecond postpulse with 20-ps delay will interact with a microplasma created by nonlinear ionization in the focal region at near its maximum free-carrier density of $\approx 10^{19} \mathrm{~cm}^{-3}$ according the measured nanosecond time scale for electron diffusion and recombination [16]. This knowledge allows us to discuss the differences in responses on the basis of plasma affects. We 


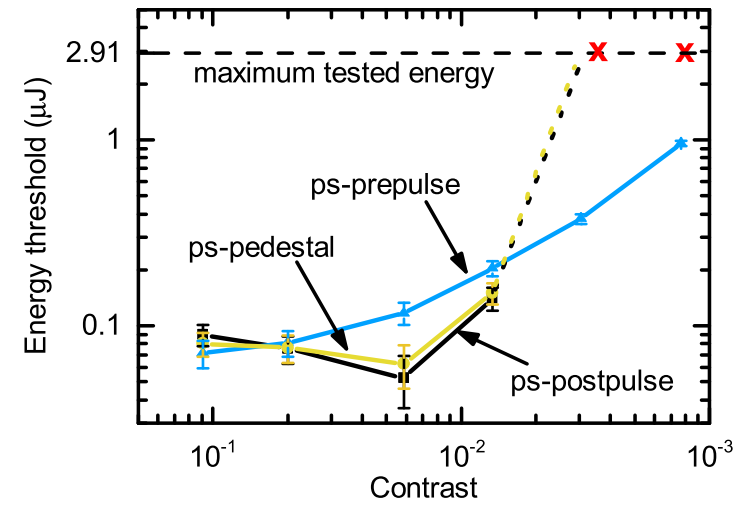

FIG. 7. Measured energy threshold for ultrafast laser induced modification in bulk silicon as function of pedestal contrast (yellow) and comparisons with satellite pre- and postpulse cases in blue and black respectively. The contrast dependence evidences a general dominating postpulse contribution in energy deposition. The red crosses represent no modification with the maximum tested energy.

note in particular in Fig. 6 that we observe more amplitude in the modulation of the measured thresholds in comparison to the picosecond prepulse case. As for prepulse, adding a postpulse can lower or increase the threshold in comparison to the pure picosecond case depending on the contrast but on a much larger extend. In other words, the sensitivity to the contrast is greatly enhanced in case of preionization seeding by the femtosecond pulse. In Fig. 6, we see a minimum for the threshold for modification with a contrast of $\approx 5 \times 10^{-2}$ that indicates likely an optimum femtosecond laser energy for efficient microplasma seeded energy deposition with the postpulse. Interestingly, the same conditions seem to create features even smaller (near threshold) than those shown in Fig. 4. This is taken as another evidence that the plasma created by the femtosecond pulse is able under certain conditions to reshape advantageously the energy flux. However, we note also that at $8 \times 10^{-3}$ the contrast is already too high to achieve modification which was not the case with prepulse. With a strong femtosecond component, the conditions turn to detrimental as it was previously observed through selfdelocalization [3-5]. According to the microplasma response, we attribute these cross-screening effects to the long-lived microsplasmas in silicon. By the same way a feedback effect of the microplasma affects the femtosecond pulse that creates it $[5,6]$, it is natural to expect similar effects cross-screening the picosecond pulse at 20-ps delay.

While the picosecond contrast is a typical issue attracting attention for ultrafast laser developments, the available information for most cases listed in Table I does not allow to access the detailed imperfections. Also for a more complete analysis, we have repeated the measurement using perfectly synchronized pulses so that we simulate the case of a picosecond pedestal centered on the main femtosecond pulse. The results are shown in Fig. 7 with direct comparison to the satellite pre- and postpulse cases discussed above. As expected, we observe a response between the pre- and postpulse cases as the picosecond pulse now contribute on both side to the femtosecond pulse. However, it is striking to note that the measured thresholds are very close to those

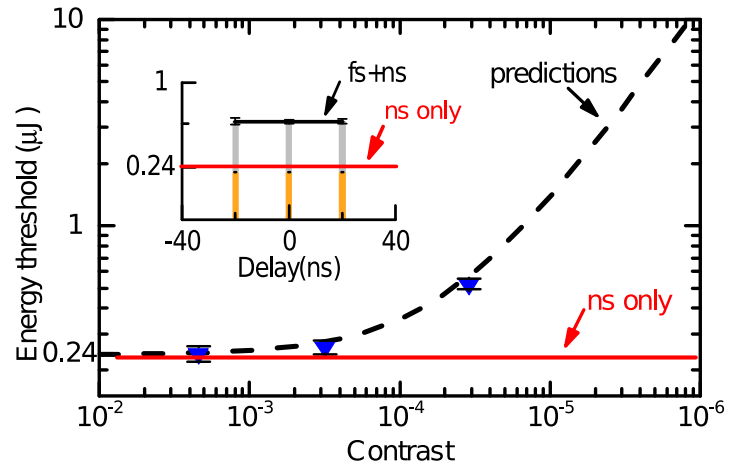

FIG. 8. Energy thresholds for ultrafast laser-induced modification in bulk silicon as function of nanosecond contrast. The contrast is given by the peak intensity ratio of a main 190-ps pulse and a synchronized 5-ns pulse. As reference the red line correspond to threshold for modification in the 'pure' nanosecond case. Comparison of the measurement(marked by blue triangle) to predictions(marked by black dashed line) assuming the absence of contribution from the femtosecond pulse indicates nanosecond background driven modification and negligible cross-action between components independently from the delay as shown by the inserted graph.

measured for the postpulse case. This can be taken as direct evidence of stronger cross-acting effects between pulses with microplasma generated when the femtosecond pulse is first.

Despite the measurable differences between these cases, a first conclusion is that typical contrast values of the emerging infrared ultrafast laser technologies can lead to permanent modifications in silicon that would be inaccessible with perfectly contrasted femtosecond pulses. A threshold is found at different contrast-dependent energy levels with a large and dominating contribution from the picosecond satellite energy distribution. We note that submicrojoule threshold energies are measured in all cases for contrasts in the range $10^{-2}-10^{-1}$. This is a typical contrast value for fiber lasers and so very consistent with the successful waveguide writing demonstrations made at similar energy level with this technology $[9,10]$ (see also Table I). It is also important to highlight here that the successfully obtained modifications with solid-state technologies have been made with pulse energies exceeding tens of microjoules [7]. This compares also very well with our analysis as it corresponds to threshold values that can be directly extrapolated from our measurements at contrast values of $\approx 10^{-3}$ or above.

Finally, we have concentrated on a picosecond contrast analysis because of the remaining challenges for a reliable ultrafast laser 3D writing solution applicable in silicon and narrow gap materials. However, as we discussed in previous section, another typical contrast imperfection for ultrafast laser technologies corresponds to an intensity background which consists of a nanosecond ASE pedestal. To simulate this case, we have repeated the investigations by electrical synchronisation of the 5-ns laser source with the femtosecond laser. We first set the synchronization to zero delay for symmetric background. The measured thresholds for modification as function of contrast are shown in Fig. 8. An important observation here is that we observe that the addition of the pedestal makes again possible to achieve modifications with 
this long-scale component while self-screening effects prevent to achieve modification with the same single femtosecond component independently of its energy. It is also noticeable that the required pulse energy to induce modification does not exceed the microjoule for contrast values down to $10^{-5}$. Then, for direct evaluation of the relative contributions of both components, we compare the measurements to a simple prediction under the hypothesis that only the nanosecond laser would contribute to the observed damage. This leads to a linear dependence to the contrast as shown by the black dashed line in Fig. 8 (shown in logarithmic scale). We make this hypothesis first for simplification but also because the nanosecond regime is known as highly favorable for achieving damage in the bulk thanks to progressive thermal bandgap closure and runway of energy deposition up to material melting [25-28]. Obviously for low contrasts the prediction tends toward the measured threshold for the pure nanosecond case shown by the red level. However, more interestingly, we also see that all our measurements are in excellent agreement with these estimates revealing in this way a negligible contribution of the femtosecond component. There is neither a significant assistance nor a cross-screening effect affecting the threshold measurements. This holds despite the large underdense plasma that inevitably develops in the prefocal region at the high femtosecond pulse energies reached in this experiment [5]. A justification is the duration of the pulse which exceeds significantly the typical microplasma lifetimes ( $<2.5 \mathrm{~ns}[16])$ measured with similarly focused femtosecond pulses. It is also confirmed by repeating the measurement at a fixed contrast of $\approx 2.8 \times 10^{-4}$ while varying the delay between components from -20 to $20 \mathrm{~ns}$ with no detectable change in the response as shown with the insert in Fig. 8.

While a negligible contribution of the femtosecond pulse in the nanosecond contrast analysis of the threshold may first sound disappointing, it represents a result of major importance for a complete comprehensive review of the literature on the topic. This is because despite threshold values similar to those of the picosecond cases, we cannot expect in this situation a major difference in the type of modification in comparison to the pure nanosecond regime. This is an aspect which actually represents a strong motivation to investigate the challenging ultrafast regime for accessing new types of modifications and improved controllability in comparison long pulse regimes (see Table I). Even in those cases where the intensity contrast would be excellent reaching $10^{-7}$ or more as those required for some high-intensity physics experiments [29,30] to avoid detrimental pre-plasma formation, we extrapolate from this analysis that the threshold for modification will be found for energies at submillijoule level. This remains relatively moderate in comparison to some previous attempts (including ours, see Table I) but the most important is that the potential benefit from the ultrafast nature of material transformations is ruined under such conditions.

\section{CONCLUSIONS}

In this paper, we have reviewed the recent but rapidly growing literature on ultrafast laser writing inside bulk silicon.
Obvious contradictions existed so far between the identified experimental windows to exceed the abnormally high modification threshold intrinsic to narrow gap materials. By synchronizing, individually controlled femtosecond, picosecond, and nanosecond pulses, we show a high sensitivity of the material response to the picosecond contrast because of the balance between detrimental or benificial preionization effects depending on the density, localization and timing of the created underdense microplamas. The analysis lead to the conclusion that the conditions for achieving bulk modifications are technology-dependent as all types of near-infrared sources are not equally performing on the temporal contrast aspect. Interestingly, the absence of anomaly in the contrast response solves all apparent contradictions.

To evaluate the consequences of this finding, it is important to note that the conclusion holds for high intensity contrast of $10^{-6}$ or even less when a thermal runaway by the nanosecond time-scale characteristic of imperfections of amplified solid-state laser technologies are considered. Accordingly, this parameter which was totally ignored in previous works should not be overlooked anymore in any future studies unless specific contrast enhancement methods as those used in strong-field physics experiments are implemented [29,30]. One method among others is to rely on nonlinear wavelength conversion (e.g., second harmonic) to generate cleaner ultrashort pulses [29]. Interestingly, this is actually intrinsically applied when OPA is used to access the appropriate infrared region of the spectrum for bulk processing in silicon. However, when making this comparison one should not ignore a major difference. In strong-field interaction experiments, the main motivation of preparing highly contrasted ultrashort pulses is actually to enhance the conditions in matter which is exactly an opposite response to what is found for ultrafast laser processing in bulk silicon.

Finally, another important conclusion is the systematic existence of an optimum temporal contrast for achieving modifications with a minimal energy budget in all investigated cases. With such a level of sensitivity to the multiscale temporal characteristics of irradiation, we anticipate temporal shaping of femtosecond pulses driver must represent another key, as hyper-focusing [5] for highly contrasted pulses, to open the door to new applications based on ultrasfast laser writing in narrow gap semiconductors. Among those applications that will not be possible otherwise, we can mention refractive index engineering in silicon giving the possibility to move silicon photonics from 2D systems to monolithic 3D architectures, the access of microexplosion regimes in semiconductors that will enable the study of new dense phases of solids and precision surgery of tissues or biological substance that are opaque to visible (or NIR) light.

\section{ACKNOWLEDGMENTS}

We thank Raphael Clady and engineers of Light Conversion for fruitful discussions on the laser technology aspects. This research has received funding from the European $\mathrm{Re}$ search Council (ERC) under the European Union's Horizon 2020 research and innovation program (Grant Agreement No. 724480). 
[1] R. R. Gattass and E. Mazur, Femtosecond laser micromachining in transparent materials, Nat. Photonics 2, 219 (2008).

[2] K. Sugioka and Y. Cheng, Femtosecond laser three-dimensional micro- and nanofabrication, Appl. Phys. Rev. 1, 041303 (2014).

[3] V. V. Kononenko, V. V. Konov, and E. M. Dianov, Delocalization of femtosecond radiation in silicon, Opt. Lett. 37, 3369 (2012).

[4] A. Mouskeftaras, A. V. Rode, R. Clady, M. Sentis, O. Utéza, and D. Grojo, Self-limited underdense microplasmas in bulk silicon induced by ultrashort laser pulses, Appl. Phys. Lett. 105, 191103 (2014).

[5] M. Chanal, V. Y. Fedorov, M. Chambonneau, R. Clady, S. Tzortzakis, and D. Grojo, Crossing the threshold of ultrafast laser writing in bulk silicon, Nat. Commun. 8, 773 (2017).

[6] M. Chambonneau, L. Lavoute, D. Gaponov, V. Y. Fedorov, A. Hideur, S. Février, S. Tzortzakis, O. Utéza, and D. Grojo, Competing Nonlinear Delocalization of Light for Laser Inscription Inside Silicon with a 2- $\mu \mathrm{m}$ Picosecond Laser, Phys. Rev. Appl. 12, 024009 (2019).

[7] M. Mori, Y. Shimotsuma, T. Sei, M. Sakakura, K. Miura, and H. Udono, Tailoring thermoelectric properties of nanostructured crystal silicon fabricated by infrared femtosecond laser direct writing, Phys. Status Solidi A 212, 715 (2015).

[8] H. Kämmer, G. Matthäus, S. Nolte, M. Chanal, O. Utéza, and D. Grojo, In-volume structuring of silicon using picosecond laser pulses, Appl. Phys. A-Mater. 124, 302 (2018).

[9] I. Pavlov, O. Tokel, S. Pavlova, V. Kadan, G. Makey, A. Turnali, Ö. Yavuz, and F. Ö. Ilday, Femtosecond laser written waveguides deep inside silicon, Opt. Lett. 42, 3028 (2017).

[10] G. Matthäus, H. Kämmer, K. A. Lammers, C. Vetter, W. Watanabe, and S. Nolte, Inscription of silicon waveguides using picosecond pulses, Opt. Express 26, 24089 (2018).

[11] A. H. Nejadmalayeri, P. R. Herman, J. Burghoff, M. Will, S. Nolte, and A. Tünnermann, Inscription of optical waveguides in crystalline silicon by mid-infrared femtosecond laser pulses, Opt. Lett. 30, 964 (2005).

[12] Y. Ishikawa, Y. Shimotsuma, A. Kaneta, M. Sakakura, M. Nishi, K. Miura, K. Hirao, and Y. Kawakami, Fabrication of photoinduced microstructure embedded inside $\mathrm{ZnO}$ crystal, Proc. SPIE 8243, 82430N (2012).

[13] V. V. P. Sreenivas, M. Bülters, and R. B. Bergmann, Microsized subsurface modification of mono-crystalline silicon via nonlinear absorption, J. Eur. Opt. Soc.-Rapid 7, 12035 (2012).

[14] D. Grojo, S. Leyder, P. Delaporte, W. Marine, M. Sentis, and O. Utéza, Long-wavelength multiphoton ionization inside bandgap solids, Phys. Rev. B 88, 195135 (2013).

[15] S. Leyder, D. Grojo, P. Delaporte, W. Marine, M. Sentis, and O. Utéza, Non-linear absorption of focused femtosecond laser pulses at $1.3 \mu \mathrm{m}$ inside silicon: Independence on doping concentration, Appl. Surf. Sci. 278, 13 (2013).

[16] A. Mouskeftaras, M. Chanal, M. Chambonneau, R. Clady, O. Utéza, and D. Grojo, Direct measurement of ambipolar diffusion in bulk silicon by ultrafast infrared imaging of laserinduced microplasmas, Appl. Phys. Lett. 108, 041107 (2016).
[17] D. Grojo, A. Mouskeftaras, P. Delaporte, and S. Lei, Limitations to laser machining of silicon using femtosecond micro-Bessel beams in the infrared, J. Appl. Phys. 117, 153105 (2015).

[18] S. Mitra, M. Chanal, R. Clady, A. Mouskeftaras, and D. Grojo, Millijoule femtosecond micro-Bessel beams for ultra-high aspect ratio machining, Appl. Optics 54, 7358 (2015).

[19] E. V. Zavedeev, V. V. Kononenko, and V. I. Konov, Delocalization of femtosecond laser radiation in crystalline $\mathrm{Si}$ in the mid-IR range, Laser Phys. 26, 016101 (2016).

[20] E. I. Mareev, K. V. Lvov, B. V. Rumiantsev, E. A. Migal, I. D. Novikov, S. Y. Stremoukhov, and F. V. Potemkin, Effect of pulse duration on the energy delivery under nonlinear propagation of tightly focused $\mathrm{Cr}$ :forsterite laser radiation in bulk silicon, Laser Phys. Lett. 17, 15402 (2019).

[21] A. Wang, A. Das, and D. Grojo, Ultrafast laser writing deep inside silicon with THz-repetition-rate trains of pulses, Research 2020, 8149764 (2020).

[22] M. Mielke, D. Gaudiosi, K. Kim, M. Greenberg, X. Gu, R. Cline, X. Peng, M. Slovick, N. Allen, M. Manning et al., Ultrafast fiber laser platform for advanced materials processing, J. Laser Micro/Nanoeng. 5, 53 (2010).

[23] Y. Xu, J. Wang, Y. Huang, Y. Li, X. Lu, and Y. Leng, Nonlinear temporal pulse cleaning techniques and application, High Power Laser Sci. Eng. 1, 98 (2013).

[24] A. Das, A. Wang, O. Uteza, and D. Grojo, Pulse duration dependence of laser-induced modifications inside silicon (unpublished).

[25] P. C. Verburg, G. R. B. E. Römer, and A. J. Huis in 't Veld, Two-photon-induced internal modification of silicon by erbiumdoped fiber laser, Opt. Express 22, 21958 (2014).

[26] M. Chambonneau, Q. Li, M. Chanal, N. Sanner, and D. Grojo, Writing waveguides inside monolithic crystalline silicon with nanosecond laser pulses, Opt. Lett. 41, 4875 (2016).

[27] X. Yu, X. Wang, M. Chanal, C. A. Trallero-Herrero, D. Grojo, and S. Lei, Internal modification of intrinsic and doped silicon using infrared nanosecond laser, Appl. Phys. A Mater. 122, 1 (2016).

[28] O. Tokel, A. Turnall, G. Makey, P. Elahi, T. ÇolakoÇlu, E. Ergeçen, Ö. Yavuz, R. Hübner, M. Zolfaghari Borra, I. Pavlov, A. Bek, R. Turan, D. K. Kesim, S. Tozburun, S. Ilday, and F. Ö. Ilday, In-chip microstructures and photonic devices fabricated by nonlinear laser lithography deep inside silicon, Nat. Photonics 11, 639 (2017).

[29] L. M. Chen, M. Kando, M. H. Xu, Y. T. Li, J. Koga, M. Chen, H. Xu, X. H. Yuan, Q. L. Dong, Z. M. Sheng, S. V. Bulanov, Y. Kato, J. Zhang, and T. Tajima, Study of X-ray Emission Enhancement via a High-Contrast Femtosecond Laser Interacting with a Solid Foil, Phys. Rev. Lett. 100, 045004 (2008).

[30] Y. Azamoum, V. Tcheremiskine, R. Clady, A. Ferré, L. Charmasson, O. Utéza, and M. Sentis, Impact of the pulse contrast ratio on molybdenum $\mathrm{K} \alpha$ generation by ultrahigh intensity femtosecond laser solid interaction, Sci. Rep. 8, 4119 (2018). 